\title{
Effects of Age and Type of Stimulus on the Cortical Auditory Evoked Potential in Healthy Malaysian Children
}

\author{
Siti Zamratol-Mai Sarah Mukari ${ }^{1}$, Cila Umat ${ }^{2}$, Soon Chien Chan ${ }^{2}$, \\ Akmaliza Ali $^{2}$, Nashrah Maamor ${ }^{2}$, and Mohd Normani Zakaria ${ }^{3}$ \\ ${ }^{1}$ Institute of Ear, Hearing and Speech, Universiti Kebangsaan Malaysia, Jalan Raja Muda Abdul Aziz, Kuala Lumpur, Malaysia \\ ${ }^{2}$ Department of Audiology and Speech Sciences, Faculty of Allied Health Sciences, Universiti Kebangsaan Malaysia, \\ Jalan Raja Muda Abdul Aziz, Kuala Lumpur, Malaysia \\ ${ }^{3}$ Audiology and Speech Pathology Programme, School of Health Sciences, Universiti Sains Malaysia, Kubang Kerian, Kelantan, Malaysia
}

Received July 1,2019

Revised October 26, 2019

Accepted December 1, 2019

Address for correspondence

Mohd Normani Zakaria, PhD

Audiology and Speech Pathology

Programme, School of Health Sciences,

Universiti Sains Malaysia,

16150 Kubang Kerian, Kelantan,

Malaysia

Tel +6097677691

Fax +6097677515

E-mail mdnorman@usm.my
Background and Objectives: The cortical auditory evoked potential (CAEP) is a useful objective test for diagnosing hearing loss and auditory disorders. Prior to its clinical applications in the pediatric population, the possible influences of fundamental variables on the CAEP should be studied. The aim of the present study was to determine the effects of age and type of stimulus on the CAEP waveforms. Subjects and Methods: Thirty-five healthy Malaysian children aged 4 to 12 years participated in this repeated-measures study. The CAEP waveforms were recorded from each child using a $1 \mathrm{kHz}$ tone burst and the speech syllable /ba/. Latencies and amplitudes of P1, N1, and P2 peaks were analyzed accordingly. Results: Significant negative correlations were found between age and speech-evoked CAEP latency for each peak $p<$ 0.05). However, no significant correlations were found between age and tone-evoked CAEP amplitudes and latencies ( $p>0.05)$. The speech syllable $/ \mathrm{ba} /$ produced a higher mean P1 amplitude than the $1 \mathrm{kHz}$ tone burst $(p=0.001)$. Conclusions: The CAEP latencies recorded with the speech syllable became shorter with age. While both tone-burst and speech stimuli were appropriate for recording the CAEP, significantly bigger amplitudes were found in speech-evoked CAEP. The preliminary normative CAEP data provided in the present study may be beneficial for clinical and research applications in Malaysian children.

J Audiol Otol 2020;24(1):35-39

KEY WORDS: Evoked potential $\cdot$ Cortical $\cdot$ Age $\cdot$ Tone burst $\cdot$ Speech.

\section{Introduction}

The ability to communicate effectively with others is made possible by an intact auditory mechanism. The importance of having a normal hearing is even more crucial for children who are in the process of developing their speech and language. Physiologically, both the peripheral and central auditory systems are involved in processing the auditory inputs. If the organs within the auditory system are disrupted, hearing loss occurs [1]. To limit the negative consequences of hearing loss, hearing assessments should be conducted as soon as possible so that those with hearing loss can undergo

This is an Open Access article distributed under the terms of the Creative Commons Attribution Non-Commercial License (https://creativecommons.org/licenses/by-nc/4.0/) which permits unrestricted non-commercial use, distribution, and reproduction in any medium, provided the original work is properly cited. appropriate interventions in a timely manner [2,3]. In fact, hearing-impaired children who received early interventions demonstrated better speech improvements than those who received late interventions $[1,3]$.

The auditory status of an individual can be determined using subjective or objective tests. Pure tone audiometry (PTA) is an example of a known subjective test for the diagnosis of hearing disorders [1]. In this test, the hearing levels at specific frequencies are obtained by asking the individual to behaviorally indicate his/her responses when specific sounds are presented. Nevertheless, objective tests are more appropriate when testing difficult-to-test children as their cooperation (in giving subjective responses) is not required.

The cortical auditory evoked potential (CAEP) is a useful objective test to measure the function of auditory organs within the central auditory nervous system $[1,4]$. It is a com- 


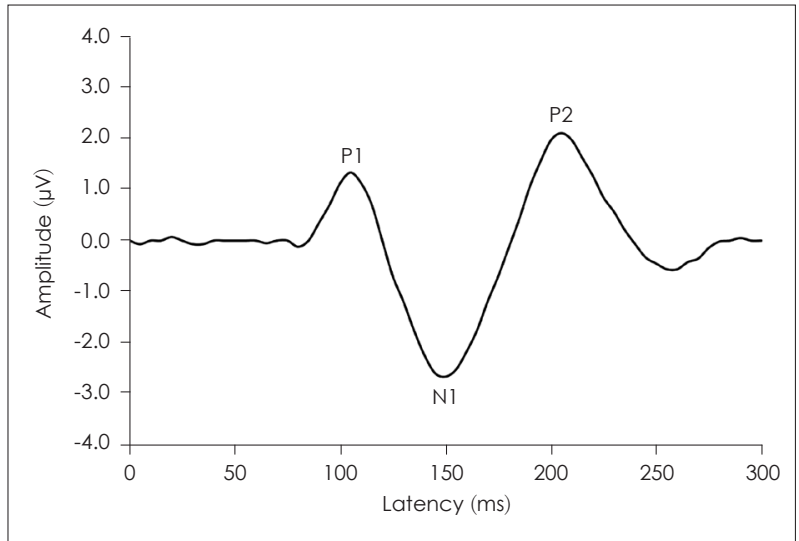

Fig. 1. A typical cortical auditory evoked potential waveform in a healthy suject (the prominent peaks are clearly shown).

ponent of the auditory evoked potential (AEP) classification based on its' peak latencies [4]. The obligatory CAEP represents the neural detection of the evoking auditory stimuli and is characterized by a series of prominent peaks ( $\mathrm{P} 1, \mathrm{~N} 1$, and P2) typically occurring within 50-300 ms (Fig. 1).

To record the CAEP, scalp electrodes are placed on specific locations of the head and stimuli such as tone bursts or speech syllables are presented repetitively to evoke electrical potentials along the central auditory pathway. As reported elsewhere, CAEP has been found to be sensitive in identifying hearing loss and disorders affecting the central auditory pathway including tumors, multiple sclerosis, and brain injury [5-7]. Abnormal CAEP waveforms have also been reported in children with syndromic features, attention deficit hyperactivity disorder, autism, learning disabilities, auditory processing disorder, auditory neuropathy, and epilepsy [8-15].

Before a specific test can be applied in clinical settings, its normative data must be established. To our knowledge, normative CAEP data for Malaysian children have not been published. However, prior to the development of the normative data for the CAEP, the possible influence of fundamental variables such as age and type of stimulus on the CAEP should be studied. Therefore, the aim of the present study was to determine the effects of age and stimulus type on the CAEP waveforms. Subsequently, the normative CAEP data for Malaysian children would be established.

\section{Subjects and Methods}

\section{Participants}

In this study, which employed a repeated-measures design, thirty-five Malaysian children aged between 4 and 12 years (mean $=8.4 \pm 2.7$ years) were recruited. They were all healthy and had no hearing, speech, or learning disabilities (their PTA thresholds were less than $25 \mathrm{~dB}$ HL at frequencies of 0.25 to $8 \mathrm{kHz}$ ). The study procedure was approved by the Institutional Review Board (FF-108-200), which is in accordance with the 1975 Declaration of Helsinki and its later amendments. Prior to the data collection, written informed consent was obtained from the caregiver of each child.

\section{Test equipment and procedures}

A two-channel Biologic AEP device (version 6.2 .0 by Natus Medical Inc., Pleasanton, CA, USA) was used to record the CAEP. The active electrode was placed on the earlobe, the indifference electrodes were placed on $\mathrm{C} 3$ and $\mathrm{C} 4$, and the reference electrode was placed on the forehead. These electrode sites were chosen to produce optimal CAEP waveforms [4]. To ensure good recording quality, the electrode impedance was maintained below $5 \mathrm{k} \Omega$ throughout the recording sessions.

During the testing, the children were seated comfortably facing a loud speaker positioned at a one meter distance at $0^{\circ}$ azimuth. They were instructed to relax and minimize their body movements, while watching a voiceless movie of their choice. The CAEP waveforms were recorded using a $1 \mathrm{kHz}$ tone burst and with the speech syllable /ba/ transmitted at an intensity level of $65 \mathrm{~dB}$ SPL. The tone burst was ramped using a Blackman filter with a 30-cycle rise/fall time and a 10-cycle plateau time. The speech syllable was custom built using a Sound Forge software (version 8.0 by Sony, New York City, NY, USA). The repetition rate for both stimuli was $1 / \mathrm{s}$. The time window was set to $533 \mathrm{~ms}$ with 512 points. The waveforms were amplified 50,000 times and band-passed at 1-30 $\mathrm{Hz}$. The artifact rejection level was set at $95 \mu \mathrm{v}$ to eliminate movements and biological interference. Breaks were given between the sessions or as requested by the subjects.

\section{Data analysis}

The prominent peaks of the CAEP were identified and plotted by experienced researchers. The latencies and amplitudes of the P1, N1, and P2 peaks were computed for each subject. Since the data were found to be normally distributed ( $p>0.05$ by Kolmogorov-Smirnov test), parametric analyses could be conducted. Pearson correlation analysis was used to determine the effect of age on the CAEP results. A paired t-test was employed to determine the influence of stimulus type on the CAEP waveforms. The statistical significance level was set at $p<0.05$. To support the hypothesis testing results, Cohen's effect size (d) was computed. The effect sizes were interpreted as small $(\mathrm{d}=0.20)$, medium $(\mathrm{d}=0.50)$, and large $(\mathrm{d}=0.80)$ [16]. All data were analyzed using the SPSS software version 20 (IBM Corp., Armonk, NY, USA). 


\section{Results}

The CAEP waveforms were successfully recorded for all children. Based on the correlation analyses, no significant relationship was found between age and the CAEP amplitude at all peaks for both stimuli ( $p>0.05)$. Likewise, no significant correlations were found between age and tone-evoked CAEP latencies $(p>0.05)$. In contrast, for the CAEP latencies recorded using the speech syllable /ba/, significant negative correlations were found between age and P1 $(r=-0.52, p=0.001), \mathrm{N} 1$ $(r=-0.55, p=0.001)$, and P2 $(r=-0.66, p<0.001)$. That is, all peak latencies shortened with age. The correlation plot for each of these peak latencies is illustrated in Fig. 2. To further verify the age effect on the CAEP waveforms, we compared the CAEP results between the following three age groups: Group 1 (4-6 years), Group 2 (7-9 years), and Group 3 (1012 years). Consistent with the correlation analyses, one-way ANOVA found no significant differences in the CAEP am- plitudes between the age groups for both tone and speech stimuli $(p>0.05)$. The mean differences between the three age groups were also insignificant for the tone-evoked CAEP latencies $(p>0.05)$. However, when evoked by speech stimuli, children in Group 3 revealed the shortest peak latencies $(p<$ $0.05)$. The longest peak latencies were noted for those in Group $1(p<0.05)$. Based on the post-hoc analysis, significant differences in peak latencies were found between Group 1 (younger children) and Group 3 (older children) ( $p>0.05$ ). Significant mean differences were found between Group 1 and Group 2 only for P1 $(p<0.05)$ but not for N1 and P2 $(p>0.05)$. When Group 2 and Group 3 were compared, significant results were noted for N1 and P2 $(p<0.05)$. The latencies between Group 2 and Group 3 were found to be comparable to P1 ( $p>$ 0.05). Collectively, these ANOVA results were all consistent with the correlation analyses supporting the notable age effect on speech-evoked CAEP latencies.

Table 1 reveals the mean and standard deviation for each
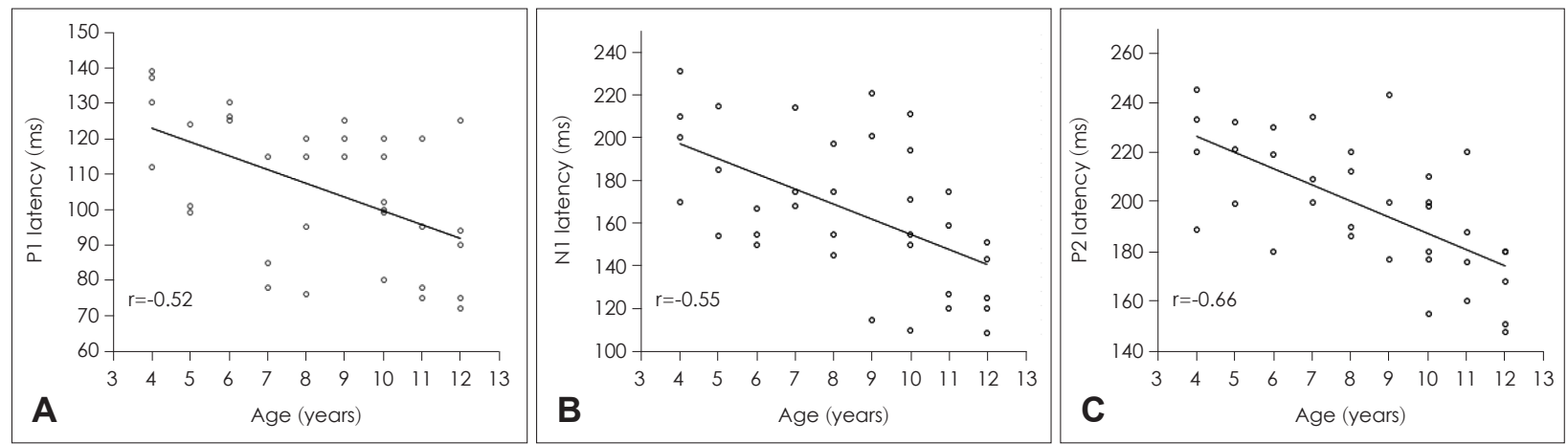

Fig. 2. Correlation plot between age and $P 1$ latency $(A), N 1$ latency $(B)$, and $P 2$ latency $(C)$.

Table 1. Mean and standard deviation of cortical auditory evoked potential (CAEP) latency for each peak when evoked by tone burst and speech stimuli

\begin{tabular}{ccccc}
\hline \multirow{2}{*}{ CAEP peak } & \multicolumn{2}{c}{ CAEP latency $($ mean \pm SD) $(\mathrm{ms})$} & \multirow{2}{*}{ p value } & Effect size $(\mathrm{d})$ \\
\cline { 2 - 5 } & Tone burst & Speech stimulus & 0.893 & 0.02 \\
P1 & $105.89 \pm 20.53$ & $105.47 \pm 13.91$ & 0.453 & 0.13 \\
P1 & $166.42 \pm 41.35$ & $171.46 \pm 33.35$ & 0.324 & 0.18 \\
\hline
\end{tabular}

The corresponding $p$ values and Cohen's effect size $(\mathrm{d})$ are also shown when the peak latencies are compared between the two stimuli

Table 2. Mean and standard deviation of cortical auditory evoked potential (CAEP) amplitude for each peak when evoked by tone burst and speech stimuli

\begin{tabular}{|c|c|c|c|c|}
\hline \multirow{2}{*}{ CAEP peak } & \multicolumn{2}{|c|}{ CAEP amplitude $($ mean \pm SD $)(\mu \mathrm{V})$} & \multirow{2}{*}{$p$ value } & \multirow{2}{*}{ Effect size $(d)$} \\
\hline & Tone burst & Speech stimulus & & \\
\hline P1 & $1.02 \pm 1.05$ & $1.89 \pm 1.29$ & $0.001^{*}$ & 0.74 \\
\hline $\mathrm{N} 1$ & $2.33 \pm 1.77$ & $2.93 \pm 1.63$ & 0.053 & 0.35 \\
\hline P2 & $1.49 \pm 1.69$ & $2.01 \pm 1.79$ & 0.096 & 0.30 \\
\hline $\mathrm{N} 1-\mathrm{P} 2$ & $3.82 \pm 2.69$ & $4.94 \pm 2.74$ & 0.089 & 0.41 \\
\hline
\end{tabular}

The corresponding p values and Cohen's effect size $(\mathrm{d})$ are also shown when the peak amplitudes are compared between the two stimuli. *Significant at $p<0.05$ in a paired t-test 
peak latency when stimulated by the tone burst and speech stimuli. As shown, no significant difference in latency was found between the tone burst and speech syllable for all peaks $(p>0.05)$. These insignificant $p$ values were further supported by the small effect sizes $(d<0.20)$. The corresponding CAEP amplitude analyses are shown in Table 2. As indicated, the speech syllable /ba/ produced a higher mean P1 amplitude than the $1 \mathrm{kHz}$ tone burst $(p=0.001)$ and the magnitude of difference was medium $(d=0.74)$. For the other peaks, no significant difference in the CAEP amplitude was noted between the two stimuli $(p>0.05)$.

\section{Discussion}

In the present study, the CAEP waveforms were recorded using tone burst and speech stimuli in Malaysian children. The CAEP latencies and amplitudes obtained are consistent with those of previous studies and are within the published ranges $[4,17,18]$. The $\mathrm{P} 1, \mathrm{~N} 1$, and $\mathrm{P} 2$ peaks of the CAEP reflect the encoding of potentially detectable sound by the neurons of the auditory cortex $[19,20]$.

As reported elsewhere, the CAEP waveforms reflect the maturation of the central auditory nervous system in their latency, amplitude, or morphology [21]. These maturational changes are complex and extend well into the second decade of life [22]. In this study, it was found that age was more related to the CAEP latencies than amplitudes, and these relationships were more predominant with the speech-evoked CAEP. Specifically, significant reductions were noted in speech-evoked P1, N1, and P2 latency. These findings are in line with previous studies where, generally, age related changes in the CAEP are associated with reduced latencies. For example, P1 latency has been reported to decline with age [22,23]. A similar pattern has also been found for N1 latency [23,24]. To obtain more insight into this age effect, the peak latencies were compared between the three age groups (i.e., 4-6, 7-9, and 10-12 years). As shown, the older children (aged $\geq 7$ years) demonstrated shorter peak latencies than younger children (aged 4-6 years), which is consistent with the existing literature [25]. However, conflicting findings have been reported for $\mathrm{P} 2$ latency. That is, while some studies revealed no age-related changes in P2 latency [26,27], others found a reduction in P2 latency with age [28]. Unlike the speechevoked CAEP outcomes, the present study revealed no significant association between age and the CAEP latencies recorded with the tone burst stimulus. This may indicate that the physiological detection of simple sounds, such as tones, could be processed with ease even in younger children.

As revealed, no significant effect of age was discovered on the CAEP amplitudes evoked by either stimulus. In the literature, contradictory findings have been reported regarding the maturational effect on the CAEP amplitudes. As such, N1 amplitude was reported to increase with age [24]. Consistent with the present study findings, no significant age effect on N1 amplitude was found in other studies [22,29].

In the present study, both simple ( $1 \mathrm{kHz}$ tone burst) and complex (speech syllable /ba/) stimuli were used to record the CAEP. The tone burst was chosen as it is a standard stimulus available in the conventional two-channel AEP device. That is, the tone-evoked CAEP can be conveniently performed by audiologists and hearing clinicians as the AEP device is readily available in many hospitals or clinics. However, the speech-evoked CAEP was studied as the speech stimulus is more natural and contains multiple frequencies [4]. As such, different findings would be expected when the CAEP is recorded with two different stimuli. As revealed, the speech syllable /ba/ produced significantly higher P1 amplitudes than the tone burst. This finding is consistent with previous reports $[4,21,30]$. In a study by Ibrahim, et al. [30], the CAEP waveforms were recorded from 15 healthy Malay adults using tone burst and speech stimuli. In line with the present study outcomes, they found that the speech stimuli produced significantly larger P1 amplitudes than the tone burst [30].

However, the CAEP latencies recorded with both stimuli were found to be comparable. This finding is sensible as the CAEP amplitudes are typically more affected than the CAEP latencies when stimuli with different frequencies are used $[4,25]$. It is worth noting that even though no significant difference was found for the N1 amplitude between the two stimuli, the $p$ value obtained was on the verge of significance $(p=0.053)$. In this regard, if more children could be recruited, more accurate statistical results can be produced. Future research with larger sample sizes is warranted to verify this possibility.

While no significant effect of age was found on the toneevoked CAEP results, the CAEP latencies recorded with the speech syllable became shorter with age. Both tone-burst and speech stimuli were appropriate for recording the CAEP. However, significantly larger P1 amplitudes were found for the speech-evoked CAEP. The preliminary normative data for the CAEP provided in the present study may be beneficial for clinical and research applications involving Malaysian children. Nevertheless, future large-scale research is recommended to further support and validate the present study findings. As such, the sensitivity and specificity of the CAEP can be determined by testing Malaysian children with specific auditory disorders. Furthermore, these normative data can also be compared with the performance of hearing-impaired chil- 
dren who use hearing aids or have cochlear implants. These aspects of CAEP are subject to further research.

\section{Acknowledgments}

This study was part of a research project funded by the Ministry of Science, Technology, and Innovation through a Science Fund (0601-02-SF0395). The authors wish to thank Syarifah Nur Ramadhani for her assistance with data collection.

\section{Conflicts of interest}

The authors have no financial conflicts of interest.

\section{Author Contributions}

Conceptualization: Siti Zamratol-Mai Sarah Mukari, Cila Umat. Data curation: Soon Chien Chan, Akmaliza Ali. Formal analysis: Mohd Normani Zakaria, Akmaliza Ali. Funding acquisition: Siti Zamratol-Mai Sarah Mukari. Investigation: Cila Umat, Soon Chien Chan. Methodology: Siti Zamratol-Mai Sarah Mukari, Akmaliza Ali. Project administration: Siti Zamratol-Mai Sarah Mukari. Software: Mohd Normani Zakaria, Nashrah Maamor. Supervision: Siti ZamratolMai Sarah Mukari, Cila Umat, Akmaliza Ali. Writing — original draft: Siti Zamratol-Mai Sarah Mukari, Cila Umat, Soon Chien Chan. Writing — review \& editing: Mohd Normani Zakaria, Nashrah Maamor.

\section{ORCID iDs}

\section{Siti Zamratol-Mai Sarah Mukari} https://orcid.org/0000-0001-5749-8928

Cila Umat Soon Chien Chan Akmaliza Ali Nashrah Maamor https://orcid.org/0000-0002-9619-4756 https://orcid.org/0000-0002-2270-6069

Mohd Normani Zakaria https://orcid.org/0000-0003-2222-952X https://orcid.org/0000-0002-6054-7089

https://orcid.org/0000-0002-3694-3460

\section{REFERENCES}

1) Katz J. Handbook of clinical audiology. 4th ed. Baltimore, USA: Williams \& Wilkins;1994. p.6-24.

2) Lieu JE. Speech-language and educational consequences of unilateral hearing loss in children. Arch Otolaryngol Head Neck Surg 2004;130:524-30.

3) Yoshinaga-Itano C, Sedey AL, Coulter DK, Mehl AL. Language of early- and later-identified children with hearing loss. Pediatrics 1998; 102:1161-71.

4) Hall JW. New handbook of auditory evoked responses. 1st ed. Boston, USA: Pearson Education;2007. p.488-517.

5) Fischer C, Bognar L, Turjman F, Villanyi E, Lapras C. Auditory early- and middle-latency evoked potentials in patients with quadrigeminal plate tumors. Neurosurgery 1994;35:45-51.

6) Jones SJ, Sprague L, Vaz Pato M. Electrophysiological evidence for a defect in the processing of temporal sound patterns in multiple sclerosis. J Neurol Neurosurg Psychiatry 2002;73:561-7.

7) Mazzini L, Zaccala M, Gareri F, Giordano A, Angelino E. Longlatency auditory-evoked potentials in severe traumatic brain injury. Arch Phys Med Rehabil 2001;82:57-65.

8) Zarchi O, Avni C, Attias J, Frisch A, Carmel M, Michaelovsky E, et al. Hyperactive auditory processing in Williams syndrome: evidence from auditory evoked potentials. Psychophysiology 2015;52:782-9.

9) Werner K, Fosi T, Boyd SG, Baldeweg T, Scott RC, Neville BG. Temporal lobe impairment in West syndrome: event-related potential evidence. Ann Neurol 2015;77:47-57.

10) Johnstone SJ, Barry RJ. Auditory event-related potentials to a two- tone discrimination paradigm in attention deficit hyperactivity disorder. Psychiatry Res 1996;64:179-92.

11) Stroganova TA, Kozunov VV, Posikera IN, Galuta IA, Gratchev VV, Orekhova EV. Abnormal pre-attentive arousal in young children with autism spectrum disorder contributes to their atypical auditory behavior: an ERP study. PLoS One 2013;8:e69100.

12) Gilley PM, Sharma A, Dorman M, Martin K. Abnormalities in central auditory maturation in children with language-based learning problems. Clin Neurophysiol 2006;117:1949-56.

13) Koravand A, Jutras B, Lassonde M. Abnormalities in cortical auditory responses in children with central auditory processing disorder. Neuroscience 2017;346:135-48.

14) Campbell JD, Cardon G, Sharma A. Clinical application of the P1 cortical auditory evoked potential biomarker in children with sensorineural hearing loss and auditory neuropathy spectrum disorder. Semin Hear 2011;32:147-55.

15) Filippini M, Boni A, Giannotta M, Pini A, Russo A, Musti MA, et al. Comparing cortical auditory processing in children with typical and atypical benign epilepsy with centrotemporal spikes: electrophysiologic evidence of the role of non-rapid eye movement sleep abnormalities. Epilepsia 2015;56:726-34.

16) Cohen J. Statistical power analysis for the behavioral sciences. 2nd ed. Hillsdale, USA: Lawrence Erlbaum Associates;1998. p.19-66.

17) Gilley PM, Sharma A, Dorman M, Martin K. Developmental changes in refractoriness of the cortical auditory evoked potential. Clin Neurophysiol 2005;116:648-57.

18) Silva LAF, Magliaro FCL, Carvalho ACM, Matas CG. Maturation of long latency auditory evoked potentials in hearing children: systematic review. Codas 2017;29:e20160107.

19) Näätänen $R$, Picton $T$. The N1 wave of the human electric and magnetic response to sound: a review and an analysis of the component structure. Psychophysiol 1987;24:375-425.

20) Hyde M. The N1 response and its applications. Audiol Neurootol 1997;2:281-307.

21) Eggermont JJ. The onset and development of auditory function: contributions of evoked potential studies. J Speech Lang Pathol Audiol 1989;13:5-16.

22) Sharma A, Kraus N, McGee TJ, Nicol TG. Developmental changes in P1 and N1 central auditory responses elicited by consonant-vowel syllables. Electroencephalogr Clin Neurophysiol 1997;104:540-5.

23) Cunningham J, Nicol T, Zecker S, Kraus N. Speech-evoked neurophysiologic responses in children with learning problems: development and behavioral correlates of perception. Ear Hear 2000;21: 554-68.

24) Bruneau N, Roux S, Guérin P, Barthélémy C, Lelord G. Temporal prominence of auditory evoked potentials (N1 wave) in 4-8 year old children. Psychophysiol 1997;34:32-8.

25) Wunderlich JL, Cone-Wesson BK, Shepherd R. Maturation of the cortical auditory evoked potential in infants and young children. Hear Res 2006;212:185-202.

26) Johnstone SJ, Barry RJ, Anderson JW, Coyle SF. Age-related changes in child and adolescent event-related potential component morphology, amplitude and latency to standard and target stimuli in an auditory oddball task. Int J Psychophysiol 1996;24:223-38.

27) Ponton CW, Eggermont JJ, Kwong B, Don M. Maturation of human central auditory system activity: evidence from multi-channel evoked potentials. Clin Neurophysiol 2000;111:220-36.

28) Oades RD, Dittman-Balcar A, Zerbin D. Development and topography of auditory event-related potentials (ERPs): mismatch and processing negativity in individuals 8-22 years of age. Psychophysiol 1997;34:677-93.

29) Gomes H, Dunn M, Ritter W, Kurtzberg D, Brattson A, Kreuze JA, et al. Spatiotemporal maturation of the central and lateral N1 components to tones. Brain Res Dev Brain Res 2001;129:147-55.

30) Ibrahim IA, Ting HN, Moghavvemi M. The effects of audio stimuli on auditory-evoked potential in normal hearing Malay adults. Int J Health Sci (Qassim) 2018;12:25-34 . 